\title{
Measurement properties and confirmatory factor analysis of the Jefferson Scale of Empathy in Italian medical students
}

\author{
Paolo Leombruni • Mariangela Di Lillo • \\ Marco Miniotti • Angelo Picardi • Guido Alessandri • \\ Chiara Sica · Francesca Zizzi • Lorys Castelli • \\ Riccardo Torta
}

Published online: 8 August 2014

(C) The Author(s) 2014. This article is published with open access at Springerlink.com

\begin{abstract}
Medical educators agree that empathy is essential for physicians' professionalism and most studies on the patient-physician relationship demonstrate that this attitude has a key role in improving clinical outcomes. Literature findings show conflicting views in defining and measuring empathy. Nevertheless, the Jefferson Scale of Empathy (JSE) is a psychometric tool now widely used. Therefore, the aim of this study was to examine psychometrics and confirm factor structure of the Italian version of the JSE in Italian medical students (JSE S-Version). During 2012, 257 second-year Italian medical students completed the JSE S-Version. Internal consistency and test-retest reliability were assessed. A confirmatory factor analysis was performed to test the factor structure. The Italian JSE S-Version showed an acceptable internal consistency $(r=0.76)$ and test-retest reliability $(r=0.72)$.
\end{abstract}

The research is attributed to the 'Rita Levi Montalcini' Department of Neuroscience of the University of Turin.

P. Leombruni · M. Miniotti $(\bowtie) \cdot$ C. Sica $\cdot$ F. Zizzi $\cdot$ R. Torta

Clinical Psychology and Psycho-Oncology Unit, 'Rita Levi Montalcini' Department of

Neuroscience, University of Turin, 15, Via Cherasco, 10126 Turin, Italy

email: mminiotti@cittadellasalute.to.it

M. Di Lillo

Azienda Ospedaliera Marche Nord, Fano, Italy

A. Picardi

Mental Health Unit, National Center of Epidemiology, Surveillance, and Health Promotion, Italian National Institute of Health, Rome, Italy

G. Alessandri

Department of Psychology, 'La Sapienza' University of Rome, Rome, Italy

L. Castelli

Department of Psychology, University of Turin, Turin, Italy 
Confirmatory factor analysis found that the factor structure proposed by the developers of the tool provides an acceptable data fit. In this sample, female medical students showed a higher mean empathy score than did males. The present study provides evidence confirming the structural validity and reliability for the Italian JSE $\mathrm{S}$-Version. Further studies are needed to confirm these findings and to explore crosscultural differences and their implications.

Keywords Empathy $\cdot$ Medical students $\cdot$ JSE

\section{Introduction}

Despite the difficulties in precisely defining empathy, almost all agree that empathy plays a key role in positive doctor-patient relationships and contributes to improvements in clinical outcomes [1]. Consequently, empathy should be an issue specifically addressed in the core curriculum of medical studies, together with communication skills. Both have to be considered essential professional characteristics for a good physician and, in general, for all helping professions [2]. Therefore, to test the effectiveness of educational programmes, it is necessary not only to define the meaning of physician empathy, but also how to measure it. In fact, despite the wide literature regarding what physician empathy is, there is still not a generally accepted clear definition of this concept.

Aring [3] and Rogers [4] and, more recently, Hojat [5] described empathy as an ability to perceive the internal frame of reference of another person and to appreciate his or her feelings.

Many researchers agree that empathy is an important attribute for the physician because it helps to better understand, and to communicate with the patient.

Hojat and colleagues postulated that physician empathy has two components: an affective and a cognitive one [1], and Mercer and Reynolds proposed an integrative approach where physician empathy can be considered both a multidimensional and a skill-based construct [6]. On the other hand, there are authors who specify that physician empathy is more a learnable professional skill than an innate personality trait [7].

Therefore, many standardized instruments have been developed $[8,9]$ to assess levels of empathy. One of the most used tools that consider the point of view of physicians is the Jefferson Scale of Empathy (JSE), created by researchers at Jefferson Medical College in the US [5, 10-12]. These authors proposed physician empathy as 'predominantly a cognitive attribute (whereas sympathy is primarily an 'affective' personal quality)' that 'involves an understanding of the inner experiences and perspectives of the patient... with a capacity to communicate this understanding' and combined with an intention to help the patient [5, 13]. The JSE was specifically developed to measure empathy among physicians and medical students to 'capture the essence of an empathic relationship in patient care situations' [5]. It has been translated and validated in several countries all over the world [13]. In Italy, it has already been tested in a sample of physicians [14] but never among medical students. 
The present effort to spread the use of the tool is important when considering the emerging conflicting results about the evolution of empathy toward the course of graduation [15] and the possible influence of cross-cultural elements in explaining at least some of the differences observed.

The present study was designed to test three research hypotheses:

(1) The Italian version of the JSE is a reliable psychometric tool and its factor structure is consistent with the model proposed by the developers of the scale;

(2) Females exceed males in the JSE score;

(3) Cross-cultural differences emerge in the JSE scores.

Subsequently, the aims of the present study are twofold:

(1) To examine the psychometrics of the Italian version of the JSE in a sample of Italian medical students by verifying its internal consistency, test-retest reliability and factor structure;

(2) To assess Italian medical students empathy level.

\section{Methods}

\section{Participants}

The research participants were undergraduate (second-year) medical students who attended the University of Turin Medical School. Ethical approval was obtained from the University of Turin Ethical Review Committee. The study was conducted in accordance with the latest version of principles of the Declaration of Helsinki.

\section{Instrument}

The JSE is a self-report paper and pencil questionnaire developed to assess medical students' attitudes toward empathic engagement in the context of patient care. It consists of 20 items, each answered on a seven-point Likert-type scale. Half of the items are positively worded (directly scored as follows: $1=$ strongly disagree, 7 = strongly agree) and half are negatively worded (inversely scored as follows: $1=$ strongly agree, $7=$ strongly disagree). The possible total score ranges from 20 to 140 . Higher values indicate more positive attitudes toward empathic patient care, hence a higher degree of empathy [13].

The original scale was developed to be administrated to medical students; it was called the Jefferson Scale of Physician Empathy and so the acronym was JSPE [10, 11]. But, due to the increasing popularity of the JSPE and the growing demand for its use in different contexts, the authors of the scale renamed it 'Jefferson Scale of Empathy' (JSE). Nowadays, the JSE can be administered (with appropriate slight modifications of some items) to physicians and other health professionals (JSE HPVersion), medical students (JSE S-Version) and students in health professions other than medicine (JSE HPS-Version) [13]. The Italian translation and the preliminary psychometrics of this tool were performed in a previous study conducted among a 
sample of Italian physicians [14] using a version designed specifically for physicians and other health professionals derived from the original JSPE [11].

For the present study, we used the JSE S-Version in its Italian translation provided by Maio and Louis, both medical education researchers of Jefferson Medical College [13].

\section{Procedure}

Questionnaires were administered to students at the beginning of their second year of medical training, after a brief description of the study during class time. To evaluate test-retest reliability of the JSE S-Version, a subsample of the participants were retested after 2 weeks. This interval was chosen as an acceptable compromise between recall effects and unwanted condition changes. Responses were confidential and collected anonymously (students identified themselves using nicknames). Each participant received an identification number when the data were entered.

\section{Statistical analyses}

SPSS for Mac, version 20.0, was used to calculate descriptive statistics, to perform the Student's $t$ test and univariate analysis of variance for examining between-group differences in the JSE S-Version scores, to calculate the internal consistency as measured by Cronbach's alpha, and to compute the test-retest reliability as measured by the intra-class correlation coefficient between scores on the first and the second administration of the questionnaire. All statistical tests were 2-tailed, with alpha set at 0.05 .

Confirmative factor analysis was conducted using Mplus 4.0 [16]. This is a form of factor analysis used to test whether observed data fit a postulated measurement model. As opposed to a theory-generating method such as exploratory factor analysis, confirmatory factor analysis is a theory-testing model, as the researcher begins with a hypothesis prior to the analysis. This model, or hypothesis, specifies which variables will be correlated with which factors and which factors are correlated. The hypothesis is based on theory, previous research, or both. Given that the predetermined model specifies the number and composition of the factors, the research is able to explicitly test hypotheses regarding the factor structure of the data. In the present study, following procedures suggested by Hojat and colleagues [11] and by Shariat and Habibi [17], we compared the fit of three models. A one-factor model (M1) in which all 20 items were forced to load on a single factor labelled 'general medical student's empathy.' An orthogonal three-factor model (M2), entailing the 'perspective taking,' 'compassionate care,' and 'standing in the patient's shoes' factors posited as uncorrelated. Finally, a three-factor oblique model (M3), entailing these last factors posited as correlated.

To deal with the ordinal nature of the data, the estimator used for the analysis was a mean- and variance-adjusted least-squares estimator (weighted least squared mean variance). Furthermore, as the normality assumption was non-tenable (Table 3), in all successive models we employed the Satorra-Bentler [18] scaled Chi square $\left(\chi^{2}\right)$ 
statistic and corrected standard errors. This correction takes into account the deviation from normality observed in the data.

All models were specified using delta parameterization [16], and their fit was evaluated by means of the following overall indexes: $\chi^{2}$ statistic, the root mean square error of approximation, the comparative fit index, and the weighted root mean square residual. Recommended cut-off points for the root mean square error of approximation are 0.08 [19] or 0.06 [20], for the comparative fit index 0.90 [21] or 0.95 [20]. For the weighted root mean square residual, the cut-off of 1.0 has moderate to strong power to detect misspecified models with acceptable Type I error [22]. Yu reported that the weighted root mean square residual, similar to the $\chi^{2}$, might be too powerful for trivial misspecification on factor covariance when sample size is large [22]. Since the factor models are truly nested models, their relative fit was compared using the scaled difference $\chi^{2}$ devised by Satorra and Bentler [23].

\section{Results}

Response rates

Among a total population $(n=390)$ of second-year medical students of the University of Turin Medical School who were invited to participate, $76.9 \%$ $(n=300)$ agreed to do so and 257 returned the correctly completed JSE questionnaire adapted for medical students (JSE S-Version), for an overall response rate of $65.9 \%$. No demographic differences were found between students who did and did not return or complete the questionnaire. All participating students voluntarily agreed to participate in the study and were properly informed about its purposes and methods. Students did not receive any incentive for their participation in the study.

\section{Demographics}

The sample considered in statistical analyses $(n=257)$ included $114(44.4 \%)$ males and $143(55.6 \%)$ females who returned the correctly completed JSE-S Version. The mean age of the overall sample was 20.6 years $(S D=20.7)$. All students were Caucasians.

JSE S-Version scores and gender differences among Italian medical students

For questionnaire completion, no time limit had been set but students took roughly 5-10 min to complete it. The mean total score of the JSE S-Version was 108.71 $(\mathrm{SD}=10.60)$. Female students scored significantly higher than male students, providing small effect size $(d=0.32)$. Tests of between-subject effects performed on the three factors of the scale showed that females scored significantly higher on the 'compassionate care' factor, while the difference was not significant for the factors 'perspective taking' and 'standing in the patient's shoes'. The between-group 
differences, despite being significant due to the large sample size, were small in absolute terms (Table 1).

Statistics at item level are available for the interested reader.

\section{Reliability}

Internal consistency was calculated by Cronbach's alpha. Reliability for the entire scale was 0.76 for the overall sample, 0.73 for males and 0.79 for females. The testretest reliability of the JSE S-Version as measured by the intra-class correlation coefficient was 0.72 (95\% CI 0.63-0.79), calculated on $153(59.5 \%)$ students (73 males, 80 females).

\section{Confirmatory factor analysis of the Italian translation of the JSE S-Version}

The model fit indices of the one-factor and the orthogonal and correlated three-factor models are reported in Table 2. Results showed that the oblique three-factor model provided an acceptable data fit, while the one-factor model and the orthogonal threefactor model revealed a poor data fit.

In Table 3, where the three models are directly compared with each other, data showed that the oblique three-factor model performed better than the two other models. The model fit improved when the residual correlation between items 17 and 19 was excluded $\left(\chi^{2}=407.55\right.$, df $=166 ; p<0.01$; comparative fit index $=0.944$, root mean square error of approximation $=0.075$, weighted root mean square residual $=0.959$ ). Under this best fitting model, correlations between latent factors were 0.24 ('perspective taking' with 'compassionate' care), 0.73 ('perspective taking' with 'standing in the patient's shoes') and 0.26 ('compassionate care' with 'standing in the patient's shoes'). Table 2 shows standardized factor loadings. With regard to the 'perspective taking' factor, all loadings were significant and ranged from 0.30 to 0.80 (mean $=0.53 ; \mathrm{SD}=0.18$ ). For the 'compassionate care' factor,

Table 1 JSE S-Version scores

\begin{tabular}{|c|c|c|c|c|c|}
\hline $\begin{array}{l}\text { JSE S-Version subscales } \\
\text { and total score }\end{array}$ & & $\begin{array}{l}\text { Sample } \\
N=257 \\
\mathrm{M} \pm \mathrm{SD}\end{array}$ & $\begin{array}{l}\text { Males } \\
N=114 \\
\mathrm{M} \pm \mathrm{SD}\end{array}$ & $\begin{array}{l}\text { Females } \\
N=143 \\
\mathrm{M} \pm \mathrm{SD}\end{array}$ & $\begin{array}{l}p \text { value } \\
\text { (males vs. } \\
\text { females) }\end{array}$ \\
\hline \multirow[t]{2}{*}{ F1. 'Perspective taking' } & m.r. $\dagger$ & $5.56 \pm 0.66$ & $5.50 \pm 0.58$ & $5.61 \pm 0.72$ & 0.187 \\
\hline & s.r. + & $55.60 \pm 6.65$ & $54.97 \pm 5.84$ & $56.11 \pm 7.21$ & 0.173 \\
\hline \multirow[t]{2}{*}{ F2. 'Compassionate care' } & m.r. $\dagger$ & $5.66 \pm 0.65$ & $5.53 \pm 0.63$ & $5.75 \pm 0.66$ & $0.007 *$ \\
\hline & s.r. $t$ & $45.25 \pm 5.22$ & $44.27 \pm 5.03$ & $46.03 \pm 5.26$ & $0.007 *$ \\
\hline \multirow{2}{*}{$\begin{array}{l}\text { F3. 'Standing in the } \\
\text { patient's shoes' }\end{array}$} & m.r. $\dagger$ & $3.92 \pm 1.24$ & $3.79 \pm 1.15$ & $4.03 \pm 1.30$ & 0.123 \\
\hline & s.r. $t$ & $7.85 \pm 2.47$ & $7.59 \pm 2.30$ & $8.06 \pm 2.59$ & 0.126 \\
\hline \multirow[t]{2}{*}{ JSE S-Version total score } & m.r. $\dagger$ & $5.44 \pm 0.53$ & $5.34 \pm 0.46$ & $5.51 \pm 0.57$ & $0.010^{*}$ \\
\hline & s.r. $t$ & $108.71 \pm 10.60$ & $106.83 \pm 9.10$ & $110.21 \pm 11.47$ & $0.011 *$ \\
\hline
\end{tabular}

$* \alpha$ set at $0.05 ; 255$ degrees of freedom; $\uparrow$ mean ratings; $\$$ sums of ratings 
Table 2 Parameter estimates from confirmatory factor analysis of the oblique three-factor model

\begin{tabular}{lllllllll}
\hline JSE S-Version items & $\mathrm{M} \pm \mathrm{SD}$ & $\mathrm{s}$ & $\mathrm{k}$ & $\mathrm{H}$ & $\mathrm{S} \&$ & $\lambda$ \\
$\mathrm{H}$
\end{tabular}

Factor 1. Perspective taking

Item 16. Physicians' understanding of the emotional status of their patients, as well as that of their families, is an important component of the physician-patient relationship

Item 13. Physicians should try to understand what is going on in their patients' minds by paying attention to their non-verbal cues and body language

Item 20. I believe that empathy is an important therapeutic factor in medical treatment

Item 15. Empathy is a therapeutic skill without which the physician's success is limited

Item 10. Patients value a physician's understanding of their feelings which is therapeutic in its own right

Item 2. Patients feel better when their physicians understand their feelings

Item 4. Understanding body language is as important as verbal communication in the physician-patient relationship

Item 9. Physicians should try to stand in their patient's shoes when providing care to them

Item 5. A physician's sense of humour contributes to a better clinical outcome

Item 17. Physicians should try to think like their patients in order to render better care

Mean loadings factor 1

Factor 2. Compassionate care

Item 11. Patients' illness can be cured only by medical treatment; therefore, physicians' emotional ties with their patients do not have a significant influence in medical or surgical treatment

Item 8. Attentiveness to patients' personal experience does not influence treatment outcomes

Item 7. Attention to patients' emotions is not important in history taking

Item 14. I believe that emotion has no place in the treatment of medical illness

Item 18. Physicians should not allow themselves to be influenced by strong personal bonds between their patients and their family members
$6.12 \pm 0.88 \quad-0.96 \quad 0.05 \quad 0.70 \quad 0.62 \quad 0.70$

$6.01 \pm 1.05 \quad-2.50 \quad 9.15 \quad 0.62 \quad 0.51 \quad 0.74$

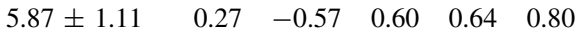

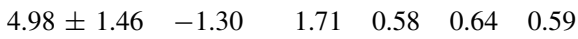

$6.00 \pm 1.05 \quad-0.39 \quad-0.60 \quad 0.58 \quad 0.58 \quad 0.60$

$6.30 \pm 1.00 \quad 0.29 \quad-0.83 \quad 0.50 \quad 0.49 \quad 0.43$

$6.07 \pm 1.07 \quad-2.50 \quad 9.20 \quad 0.46 \quad 0.54 \quad 0.49$

$5.15 \pm 1.49 \quad-1.20 \quad 1.41 \quad 0.46 \quad 0.54 \quad 0.33$

$4.64 \pm 1.59 \quad-0.65 \quad-0.22 \quad 0.45 \quad 0.44 \quad 0.30$

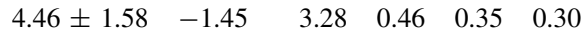

$\begin{array}{lll}0.54 & 0.53 & 0.53\end{array}$

$5.88 \pm 1.21 \quad-1.48 \quad 2.59 \quad 0.60 \quad 0.70 \quad 0.67$

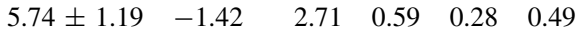

$6.46 \pm 0.87 \quad-1.29 \quad 1.94 \quad 0.55 \quad 0.59 \quad 0.70$

$6.12 \pm 1.14 \quad-1.64 \quad 2.87 \quad 0.50 \quad 0.69 \quad 0.71$

$3.27 \pm 1.44-0.45 \quad-0.51 \quad 0.44 \quad 0.06 \quad 0.04^{*}$ 
Table 2 continued

\begin{tabular}{|c|c|c|c|c|c|c|}
\hline JSE S-Version items & $\mathrm{M} \pm \mathrm{SD}$ & $\mathrm{s}$ & $\mathrm{k}$ & $\mathrm{H}$ & $\begin{array}{l}\text { S \& } \\
\mathrm{H}\end{array}$ & $\lambda$ \\
\hline $\begin{array}{l}\text { Item } 1 \text {. Physicians understanding of their } \\
\text { patients' feelings and the feelings of their } \\
\text { patients' families does not influence medical or } \\
\text { surgical treatment }\end{array}$ & $5.38 \pm 1.55$ & -1.26 & 2.89 & 0.43 & 0.36 & 0.33 \\
\hline $\begin{array}{l}\text { Item } 19 . \text { I do not enjoy reading non-medical } \\
\text { literature or experiencing the arts }\end{array}$ & $6.43 \pm 1.12$ & -0.28 & -0.65 & 0.37 & 0.40 & 0.31 \\
\hline $\begin{array}{l}\text { Item 12. Asking patients about what is happening } \\
\text { in their lives is not helpful in understanding their } \\
\text { physical complaints }\end{array}$ & $5.98 \pm 1.11$ & 0.32 & -0.45 & 0.37 & 0.53 & 0.64 \\
\hline Mean loadings factor 2 & & & & 0.48 & 0.43 & 0.49 \\
\hline \multicolumn{7}{|l|}{ Factor 3. 'Standing in the patient's shoes' } \\
\hline $\begin{array}{l}\text { Item 3. It is difficult for a physician to view } \\
\text { things from patients' perspectives }\end{array}$ & $4.07 \pm 1.35$ & -2.47 & 6.40 & 0.74 & 0.65 & 0.79 \\
\hline $\begin{array}{l}\text { Item } 6 . \text { Because people are different, it is difficult } \\
\text { to see things from patients' perspectives }\end{array}$ & $3.78 \pm 1.49$ & -1.07 & 1.08 & 0.66 & 0.55 & 0.69 \\
\hline Mean loadings factor 3 & & & & 0.70 & 0.60 & 0.74 \\
\hline
\end{tabular}

Notes All parameter estimates were significant at $p<0.05$, except item $18(*)$; the items are numbered according to Shariat \& Habibi (2012)

$s$ skewness, $k$ kurtosis, $H$ standardized factor loadings from Hojat and colleagues (2002b), $S \& H$ standardized factor loadings from Shariat \& Habibi (2012), $\lambda$ standardized factor loadings for the present study

Table 3 Goodness-of-fit indexes for the three alternative confirmatory factor analysis models

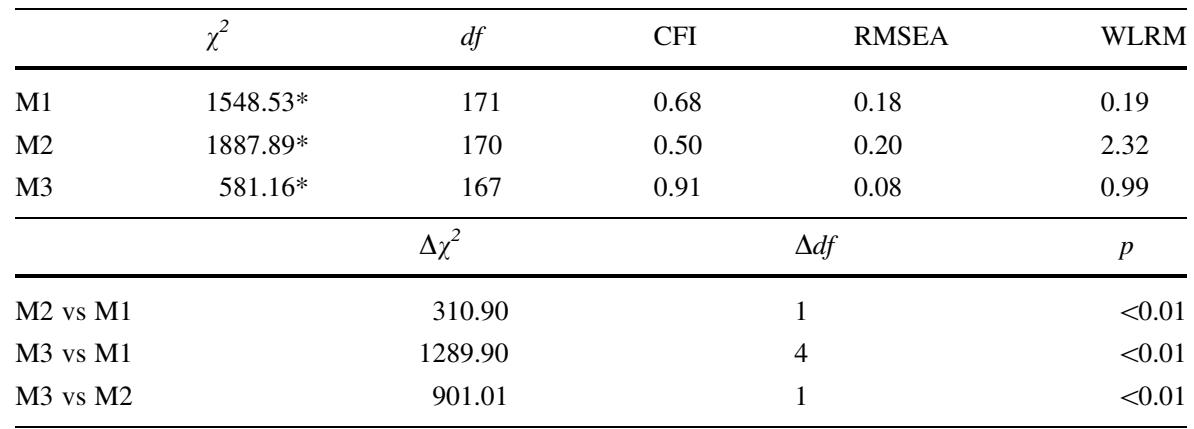

M1 one-factor model in which all 20 items were forced to load on a single factor labelled 'general medical student's empathy', $M 2$ orthogonal three-factor model entailing the 'perspective taking', 'compassionate care' and 'standing in the patient's shoes' factors posited as uncorrelated, $M 3$ oblique three-factor model entailing these last-mentioned factors posited as correlated, $\chi^{2}$ the Chi square statistic, $d f$ degrees of freedom, CFI the comparative fit index, RMSEA the root mean square error of approximation, WLRM: the weighted root mean square residual, $* p<0.01$

except the loading of item 18, all loadings were significant and ranged from 0.31 to 0.71 (mean $=0.55 ; \mathrm{SD}=0.26$ ). Finally, the two items pertaining to the 'standing in the patient's shoes' factor displayed significant and high loadings on their factor. 


\section{Discussion}

In summary, findings from the present study suggest that the Italian translation of the JSE S-Version, administered to undergraduate medical students, has satisfactory levels of internal consistency and test-retest reliability, corroborating the validity of the three-factor structure proposed by the developers of the tool [11]. Furthermore, female participants show higher levels of empathy than males.

Still regarding psychometric properties of the JSE S-Version, although its sensitivity to change needs still to be determined, its aforementioned reliability suggests that it may also have potential as an assessment tool to evaluate fluctuation in empathy over time or to verify the impact of specific training on levels of empathy.

Regarding the confirmatory factor analysis, the results of the present study showed that the oblique three-factor model suggested by the developers [11] provided an acceptable data fit, and the item loadings in the Italian sample were similar compared with the study by Hojat and his colleagues [11] and slightly higher compared with the study by Shariat and Habibi [17]. The evidence that the oblique model (where factors were allowed to be correlated) provided the best fitness could be consistent with the conceptual framework of a multidimensional notion of physician empathy. This construct seems to be composed by at least three dimensions, correlated to each other. 'Compassionate care' and 'standing in the patient's shoes' could be considered more specific to the patient-physician relationship and linked to the intention to help someone, whereas 'perspective taking' could be considered relevant in every type of human interaction [11]. Given this construct, it is reasonable to assume that the empathic behaviour of a physician could emerge from the interconnection of these three cognitive attributes.

Furthermore, while our findings, overall, corroborate the structural validity of the instrument, some factor loadings of the Italian version were found to be low (item 5 and item 17) and very low (item 18). This suggests that these items may benefit from some degree of revision, as they do not seem to function optimally.

Looking at the findings of the total mean score of empathy from the student population already studied in different countries, the total mean score of empathy of the Italian sample is significantly higher than in the UK [24], Iran [17] and Japan [25] but lower than in the USA [26]. In order to better understand and properly discuss these differences, cross-cultural comparisons should be more extensively researched. However, this is beyond the scope of the present study.

In the present study, female students showed a significant but slightly higher empathy level than males. This difference seems to be determined in particular by their higher performance on the 'compassionate care' factor. In contrast, Shariat and Habibi [17] found that their observed gender difference was mainly due to the 'perspective taking' factor. These authors noted that the negatively worded items (present in the 'perspective taking' factor and absent in the 'compassionate care' factor) could somehow have an influence in revealing assumed gender differences. In literature, unfortunately, data are inconsistent: several studies did show females to have a higher score of empathy than males, both among physicians [11, 28] and students [17, 25, 29]. A few studies did not show any differences [14, 27]. 


\section{Limitations}

The present study has some limitations that need to be underlined. First of all, the cross-sectional methodology, which gives an idea on the empathy level of the Italian students at the second year, does not allow to collect data on empathy trends. Future longitudinal studies are necessary to contribute to the current debate on the supposed 'decline' of this attitude [26]. The second relevant limitation regards the generalizability of the data. Although the demographics are representative of the Italian academic context, all the students recruited in the study belong to a single University site and fall within a single ethnic group, and this composition of the sample does not account for the possible differences among students from different regions of Italy or belonging to other ethnic groups.

\section{Conclusion}

Despite the aforementioned limitations, the present study is the first, to our knowledge, to use the Italian JSE S-Version, providing psychometric information about this tool. In particular, the results show that this measuring instrument has a satisfactory internal consistency and test-retest reliability. Moreover, findings from confirmatory factor analysis corroborate the structural validity of the oblique threefactor model suggested by the developers [11]. Regarding the empathy level of the Italian medical students, females obtained higher empathy rates than males, as emerged in previous studies [17, 25, 29]. However, further studies are needed to confirm these preliminary results, and to better explore the impact of cross-cultural differences on empathy among this population.

\section{Essentials}

- The Italian JSE S-Version seems to be a reliable psychometric instrument.

- The oblique three factor model proposed by the JSE S-Version developers seems to provide an acceptable data fit.

- The Italian JSE S-Version seems to show a structural validity; nevertheless, some items do not seem to function optimally.

- Female Italian medical students show slightly higher empathy than their male colleagues as measured by JSE S-Version.

- Italian medical students show higher empathy than UK, Iranian and Japanese medical students and lower than American as measured by JSE S-Version.

Acknowledgments The authors wish to thank Professor Mohammadreza Hojat for his suggestions about study methods and for a critical review of the manuscript.

Conflict of interest The authors report no declarations of interest. 
Ethical approval Ethical approval was obtained from the University of Turin Ethical Review Committee. More details are given in Methods section.

Funding The authors declare that no funding was received for the present study.

Open Access This article is distributed under the terms of the Creative Commons Attribution License which permits any use, distribution, and reproduction in any medium, provided the original author(s) and the source are credited.

\section{References}

1. Hojat M. Empathy in patient care. Antecedents, development, measurement, and outcomes. New York: Springer; 2007.

2. Veloski J, Hojat M. Measuring specific elements of professionalism: empathy, teamwork, and lifelong learning. In: Stern DT, editor. Measuring medical professionalism. New York: Oxford University Press; 2006.

3. Aring CD. Sympathy and empathy. J Am Med Assoc. 1958;167(4):448-52.

4. Rogers CR. A theory of therapy: personality and interpersonal relationships as developed in the clientcentered framework. In: Koch S, editor. Psychology, a study of science: foundations of the person and the social context. New York: McGraw-Hill; 1959.

5. Hojat M, Mangione S, Nasca TJ, Gonnella JS. Empathy scores in medical school and ratings of empathic behavior three years later. J Soc Psychol. 2005;145(6):663-72.

6. Mercer SW, Reynolds WJ. Empathy and quality of care. Br J Gen Pract. 2002;52(Suppl):S9-12.

7. Neumann M, Bensing J, Mercer S, Ernstmann N, Ommen O, Pfaff H. Analyzing the 'nature' and 'specific effectiveness' of clinician empathy: a theoretical overview and contribution towards a theory-based research agenda. Patient Educ Couns. 2009;74(3):339-46.

8. Hemmendinger JM, Stoddart SD, Lilford RJ. A systematic review of tests of empathy in medicine. BMC Med Educ. 2007;25:7-24.

9. Pedersen R. Empirical research on empathy in medicine-a critical review. Patient Educ Couns. 2009;76(3):307-22.

10. Hojat M, Mangione S, Nasca TJ, et al. The Jefferson Scale of Physician Empathy: development and preliminary psychometric data. Educ Psychol Meas. 2001;61:349-65.

11. Hojat M, Gonnella JS, Nasca TJ, Mangione S, Vergare M, Magee M. Physician empathy: definition, component, measurement and relationship to gender and specialty. Am J Psychiatry. 2002;159(9): 1563-9.

12. Hojat M, Erdmann JB, Gonnella JS. Personality assessment and outcomes in medical education and the practice of medicine. AMEE guide No. 79. Med Teach. 2013. doi:10.3109/0142159x.2013.785654.

13. Hojat M, Gonnella JS, Maxwell K. Jefferson Scales of Empathy (JSE). Professional manual and user's guide. Philadelphia: Jefferson Medical College; 2009.

14. Di Lillo M, Cicchetti A, Lo Scalzo A, Taroni F, Hojat M. The Jefferson Scale of Physician Empathy: preliminary psychometrics and group comparisons in Italian psysicians. Acad Med. 2009;84(9): 1198-202.

15. Neumann M, Edelhäuser F, Tauschel D, et al. Empathy decline and its reasons: a systematic review of studies with medical students and residents. Acad Med. 2011;86(8):996-1009.

16. Muthén LK, Muthén BO. Mplus user's guide. Los Angeles: Muthén \& Muthén; 2006.

17. Shariat SV, Habibi M. Empathy in Iranian medical students: measurement model of the Jefferson Scale of Empathy. Med Teach. 2013;35(1):e913-8.

18. Satorra A, Bentler PM. Scaling corrections for Chi square statistics in covariance structure analysis. In: Proceeding of the business and economics sections. Alexandria: American Statistical Association (ASA); 1988.

19. Brown MW, Cudeck R. Alternative ways of assessing model fit. In: Bollen KA, Long JS, editors. Testing structural equation models. Newbury Park: Sage; 1993.

20. Hu L, Bentler PM. Fit indices in covariance structure modelling: sensitivity to under-parameterized model misspecification. Psychol Methods. 1998;4(3):424-53. 
21. Bentler PM. Comparative fit indexes in structural models. Psychol Bull. 1990;107(2):238-46.

22. Yu CY. Evaluating cut-off criteria of model fit indices for latent variable models with binary and continuous outcomes [Doctoral dissertation]. Los Angeles: University of California; 2002.

23. Satorra A, Bentler PM. A scaled difference Chi square test statistic for moment structure analysis. Psychometrika. 2001;66(4):507-14.

24. Austin EJ, Evans P, Goldwater R, Potter V. A preliminary study of emotional intelligence, empathy and exam performance in first year medical students. Pers Individ Dif. 2005;39(5):1395-405.

25. Kataoka HU, Koide N, Ochi K, Hojat M, Gonnella JS. Measurement of empathy among Japanese medical students: psychometrics and score differences by gender and level of medical education. Acad Med. 2009;84(9):1192-7.

26. Hojat M, Vergare MJ, Maxwell K, et al. The devil is in the third year: a longitudinal study of erosion of empathy in medical school. Acad Med. 2009;84(9):1182-91.

27. Shariat SV, Keikhavani A. Empathy in residents of clinical specialties of Iran University of Medical Sciences. Iran J Psychiatry Clin Psychol. 2010;16:248-56.

28. Shariat SV, Eshtad E, Ansari S. Empathy and its correlates in Iranian physicians: a preliminary psychometric study of the Jefferson Scale of Physician Empathy. Med Teach. 2010;32(10):417-21.

29. Hojat M, Gonnella JS, Mangione S, et al. Empathy in medical students as related to academic performance, clinical competence and gender. Med Educ. 2002;36(6):522-7.

Paolo Leombruni is an assistant professor of Clinical Psychology at the University of Turin. He has published papers on psychosomatic issues (in particular, focused on eating disorders and psycho-oncology) and recently extended his research interests to medical education. He teaches in undergraduate and postgraduate courses at the University of Turin Medical School.

Mariangela Di Lillo is an internal medicine specialist at the Azienda Ospedaliera Marche Nord in Fano, Italy. Together with Professor Hojat (Research Professor at the Thomas Jefferson University of Philadelphia, developer of the Jefferson Scale of Empathy), she previously investigated the empathic behaviour among Italian physicians using the Jefferson Scale of Empathy.

Marco Miniotti is a clinical psychologist and research trainee at the Department of Neuroscience at the University of Turin. He is a tutor in the courses taught by Professors Leombuni and Torta and clerkship tutor at the Clinical Psychology and Psycho-Oncology Unit at the University of Turin.

Angelo Picardi is a researcher in the Mental Health Unit of the National Center of Epidemiology, Surveillance, and Health Promotion at the Italian National Institute of Health in Rome. He has published papers on the attachment theory and genetics of attachment and on several psychosomatic issues.

Guido Alessandri is a researcher in the Department of Psychology at the 'La Sapienza' University of Rome. He has published papers on psychological and psychometrical issues.

Chiara Sica is a physician at the Department of Neuroscience at the University of Turin.

Francesca Zizzi is a physician at the Department of Neuroscience at the University of Turin.

Lorys Castelli is a clinical psychologist, $\mathrm{PhD}$ in Neuroscience. He has published papers on neuropsychological aspects of several neurological syndromes and on psycho-oncology and clinical psychology. He teaches in undergraduate courses at the University of Turin Psychological School.

Riccardo Torta is an associate professor of Clinical Psychology and Director of Clinical Psychology and Psycho-Oncology Unit at the University of Turin. He has published papers on a number of topics, including psycho-oncology, psychopharmacology, interactions between psychotropic and chemotherapeutic drugs and psychological aspects of pain. 